\title{
An Intelligent Doctor-Patient Interaction System for Chronic Disease Telemonitoring
}

\author{
Xiang Fang, Qingfei Wang, Gen Zhu, Shu Li \\ Computer Teaching and Research Office, Hubei University of Medicine \\ Shiyan, Hubei Province, China \\ fangxiang0326@126.com
}

Keywords: Chronic disease, Telemonitoring, Mobile health, Doctor-patient interaction

\begin{abstract}
This paper proposes an instant doctor-patient interaction system for chronic disease telemonitoring. The system consists of three parts, electronic prescription, medicine reminder and intelligent alarming module. With the system doctors and patients can get the necessary information during the monitoring without face-to-face communication, which will reduce the burdens of both sides. We first introduce the working process of the whole system, and then state the modeling and implementation of three parts respectively. Primary test results show that the system can efficiently solve the problem of instant interaction between doctors and patients during telemonitoring thus improve the quality of monitoring effect.
\end{abstract}

\section{Introduction}

With the development of medicine science and technology, the lethality of infectious disease has been reduced significantly, and chronic disease is becoming the leading role of human spectrum of disease[1]. CCM(Chronic Care Model) is a Chronic disease care model based on EMA (Evidence-Based Medicine)[2] theory, and is proposed by Wagner[3] in 1996. This model reduces chronic disease patients and society health care system to some basic elements or toolkits, such as medical service support, decision support, information system, patient's self-health management, community resources, related policies and so on. By relocation and redesign for these elements, a new patient-centered medical care pattern is generated, which is especially suitable for long-term health management of chronic disease patients. Many research have shown the efficiency of model and the model is widely used in health management. The related information system is the key for the digitization and intellectualization of chronic disease care model.

Recent years many researchers were absorbed in the design of telemonitoring for health management and got quite a lot exciting results. Lin[4] proposes a wireless PDA-based physiological monitoring system, with which patients can acquire continuously their vital signals, including heart rate, three-lead electrocardiography and $\mathrm{SpO} 2$, and then transmit these signals in real-time to a remote central management unit. Chung[5] describes a cell phone based health monitoring system with self-analysis processor using wireless sensor network technology. The system utilizes a wireless dongles prototype as the intermediary devices to remotely monitor the physiological signs of patient's from a tiny wireless sensor to transmit directly to medical center monitoring/PDA wirelessly within wireless LAN or using cell phone to relay the medical data through CDMA network when outside the coverage LAN. Curtis Dorothy W[6] proposes the SMART (Scalable Medical Alert Response Technology) system, which integrates wireless patient monitoring (ECG, SPOz), geo-positioning, signal processing, targeted alerting, and a wireless interface for caregivers. Earle[7] proposes a mobile telemonitoring system for achieving tighter targets of blood pressure control in patients with complicated diabetes. They randomized 137 patients with diabetes and hypertension to either mobile telemonitoring (n-72) or usual care (n-65). Clinic blood pressure was recorded at baseline and after 6 months. The results show that systolic blood pressure fell significantly in the patients in the intervention group and remained unchanged in the control group. 
However, the healthcare systems mentioned above mainly focus on the collection, the analysis and the transmission of the physiological signals. To realize a practical telemonitoring system, we also have to consider how to get the information that was got by face-to-face communication in traditional treat. To get the alternative mechanism the following problems have to be solved.

1) Doctor sends medical advice through the information system, which is transparent to patients and they just need to follow the advice.

2) The medical advice has to be high reliability so that the patients can receive it correctly.

3) The executive condition of medical advice by the patients can be synced to the doctor workstation at any time so the doctor can understand the effect of the treat and make necessary adjustment.

4) The system should insure that the patients follow the medical advice correctly, taking drugs at right time, and start the alarm when patients don't follow the medical advice or emergency happens.

Take the factors mentioned above into consideration, we propose an intelligent doctor-patient interaction model and its implementation to solve the problem thus improve the integrity and practicability of remote medical service.

\section{Overview of the System}

The chronic disease telemonitoring system consists of four parts, the patient terminal, the doctor terminal, the nurse terminal and the database. The database in the hospital stores all the data and diagnose algorithms, the three roles in the system can operate different functions with their terminals which connect to the database through internet or private network.

The doctor-patient interaction system starts to work after the normal register when the patients go to hospital and meet the doctor for the first time and the hospital establishes related electronic record of the patient in database. Firstly the doctor send the electronic prescription based on initial diagnostic, and then patients go back home with drugs and execute the items of the prescription under the help of the drug reminder and alarming module. During the execution the system send the feedback to doctor workstation to help make necessary adjustment.

\section{Modeling and Implementation}

\section{Electronic prescription module.}

Different from tradition prescription that usually includes diagnosis and drug list, the electronic prescription integrate all the information needed during the treat process. with the automation and information technology, the prescription tries to become the interaction channel between doctors and patients even though they can't see each other. the prescription mainly includes the following information.

1) Diagnosis results. The diagnosis result is made by the doctor based on the monitoring results on patient's physiological signals.

2) Drug list. The prescription should tell the patients which kinds of drugs to take and also the information of dosage, frequency and duration time.

3) Sensors list. what kinds of physiological sensors the patient should connect to the system to get related physiological parameter such as blood pressure, blood glucose, heart rate and so on.

4) Signal collection strategy. To avoid the redundancy during continuous signal transmission, doctors will decide when to start to collect the signal, or just collect the signals in specific period which are important to diagnose.

5) The alarming threshold. Doctors set different threshold values based on different patients and different conditions. The patient terminal will give an alarm once the physiological parameters exceed the threshold values set, moreover, the alarm information will also be synced to the doctor workstation. 
A detailed prescription has a lifecycle which concludes quite a lot states. The lifecycle starts when the doctor send it to the patient terminal, and ends when the patient completes the execution or the doctor abolish it midway. The state diagram are shown in Fig.1.

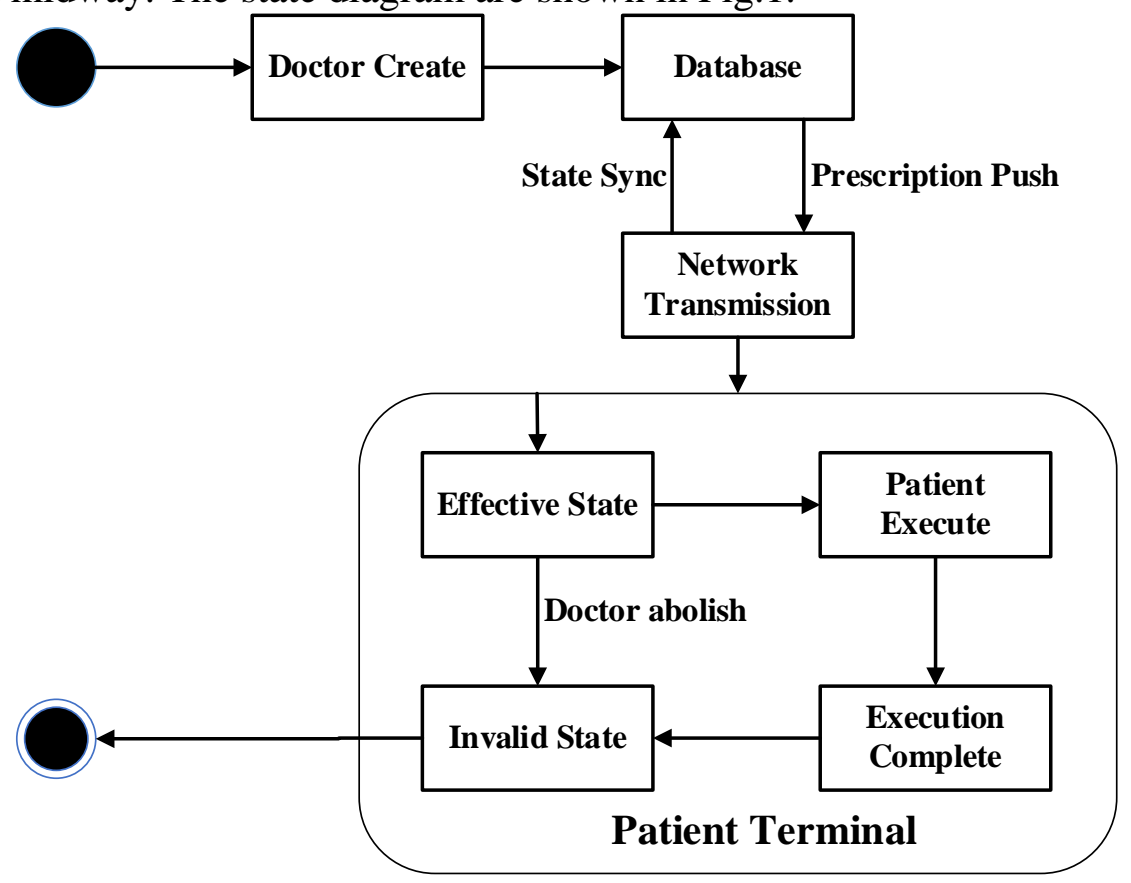

Fig. 1 The state diagram of the electronic prescription

The patient will execute acknowledgement operation on the terminal as soon as he/she has taken drugs as the prescription says. The acknowledgement will update the execution state of the prescription and sync to the doctor workstation as well. If the patient doesn't take drugs when the time indicated in the prescription arrives, the terminal will remind the patient through sound and prompt box. If the patient doesn't execute the prescription after a specific period of time, the terminal will send this condition to the doctor for further treatment.

The implementation of the electronic prescription is based on the simulation of traditional treatment process and the object-oriented design concept, Fig.2 shows the UML diagram[8].

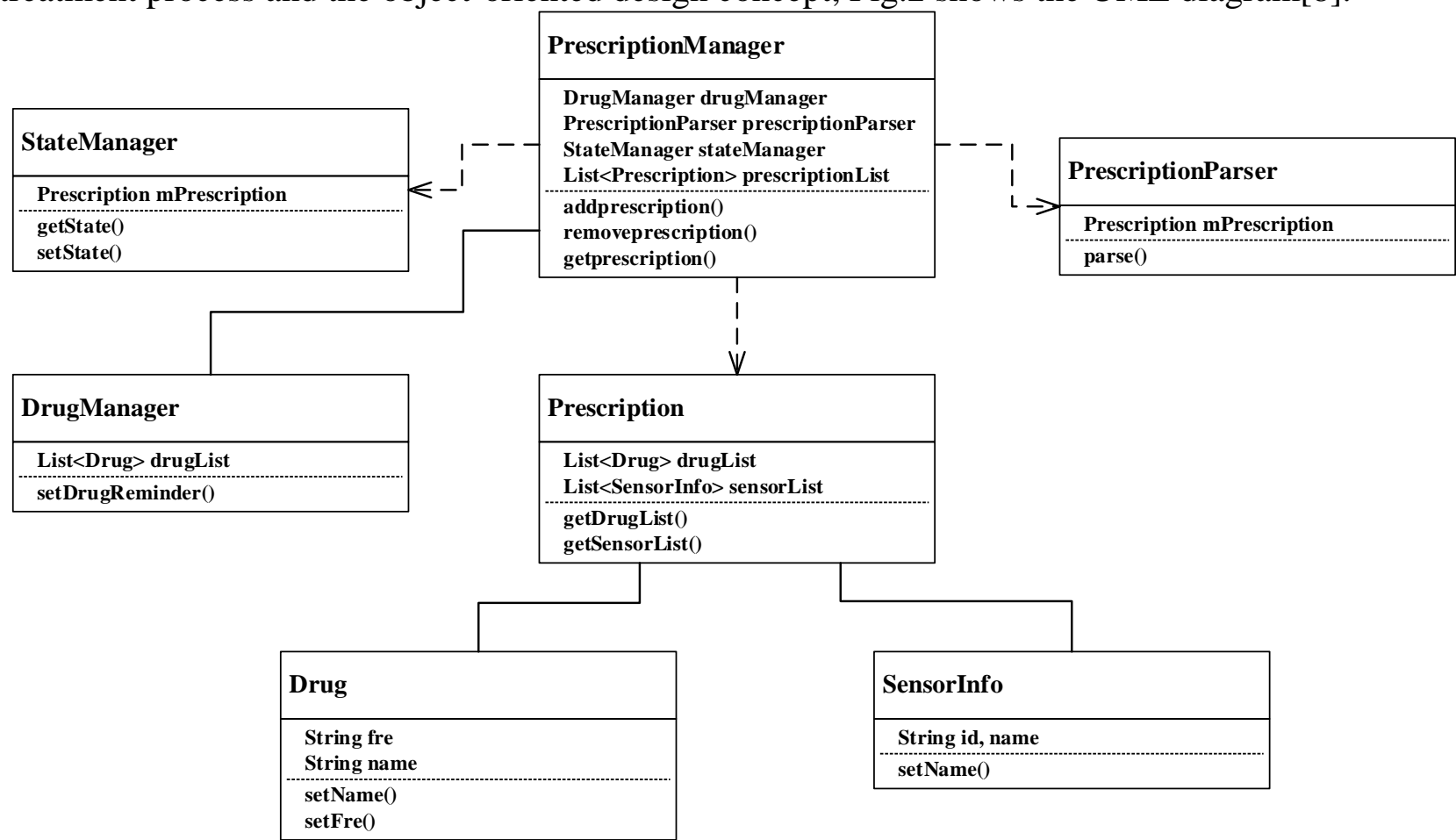

Fig. 2 The UML diagram of the electronic prescription 
The key class in the design is PrescriptionManager class, it takes charge of managing every detail of the prescription during its lifecycle. When the prescription sent by the doctor workstation is arrived, the system firstly get the prescription file through getPrescription method, and then uses PrescriptionParser class to parser the prescription file, and add the elements parsed in the prescription into prescriptionList for management. After the preparation work get ready, the system starts the DrugManager class to deal with drugs execution, the DrugManager class remind the patient to take drugs with dialog and sound signals, and also record the execution progress listed in the drugList class. PrescriptionManager class has a single instance in which it starts the StateManager thread to update the state of the prescription timely. To get the state of the prescription, the Prescription can also use the asynchronous method getState in StateManager class.

The prescription is generated by the doctor through graphical user interface on the workstation, and the workstation generated a XML file[9] based on the doctor's operation, as soon as the doctor affirms the operation, the XML file will be sent to the patient terminal through internet, the patient terminal will parse the XML file automatically when it received the file, and then notify the patient by the notification bar to view the prescription content.

\section{Drug Reminder module.}

Drug reminder management is a very important factor in chronic disease management. The chronic disease need patients take various drugs over a long period of time, and have stricter demands on time, for doctors need to collect the physiological parameter before or after the drug time to make related analysis and diagnosis. However, patients suffer from chronic disease are usually old people with a bad memory and are easy to forget the right time to take drugs thus make a negative effect on the chronic disease management. Therefore, a drug reminder module is essential in the interaction system.

The drug reminder module is design for the following purpose.

1) Automatically remind the patient to take drugs based on the time in the prescription, and also point out the kinds and dosage of related drugs.

2) When the patient has taken the drugs and confirm it on the terminal, the drug reminder module record the correct time and sync the information to the doctor workstation.

3) If the patient choose to take drugs later, the drug reminder will remind the patient again after a specific period of time, 10 minutes for example, until the patient execute the confirm operation. If the confirm operation is still undone for a long time, 2 hours for example, the drug reminder should send emergency signal to the alarming module for further dealing.

4) Both the patient and the doctor can check out the execution progress of drugs in a graphic interface on their own device.

The implementation of the drug reminder module is based on the AlarmManager class, the NotificationManager class and other Activity modules in Android SDK[10, 11]. The AlarmManager class is used to generate clock which will trigger other operations such as dialog and sound on the time set in initialization operation. The NotificationManager class is used to push important messages to the patient, for example,when new prescription is arrive and parsed, the detailed information will be pushed through the notification with prompt tone on the terminal so that the patient can read it timely.

\section{Alarming module.}

The alarming module is used to improve the flexibility and reliability of the system, and to reduce the workload of the doctor, thus make the most use of the Android terminal resources.

The alarming module will send alert information to patients and doctors under the following circumstances.

In the first circumstance the patient doesn't upload the physiological parameter in appointed time. For example, a hypertensive should connect the sensors as the prescription assigns to upload the signals, but the alarm module doesn't detect any signal at that time, then it will send alarm information to remind the patient. The whole process is shown in Fig.3. 


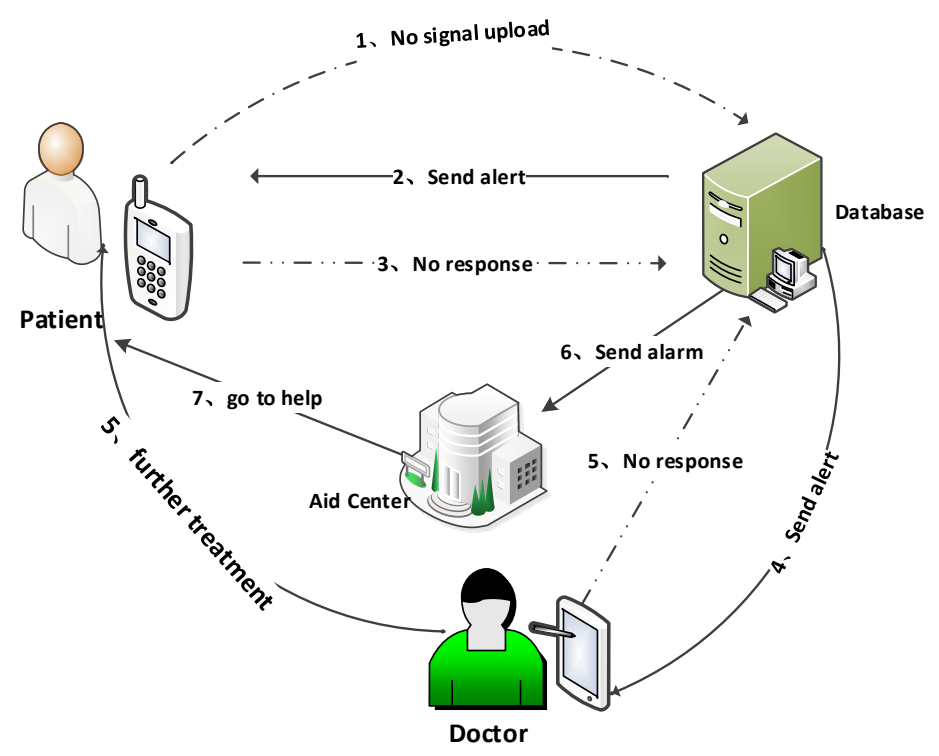

Fig. 3 the situation when the patient doesn't upload the signals

The patient doesn't upload the physiological parameter on time, which will be detected by the doctor workstation, and then the workstation send the message to the patient terminal. For some reason the patient doesn't get the reminder message or doesn't respond to it, the workstation immediately upgrade emergency level, and send the message to the nurse who is responsible for this patient, the nurse goes to the place the patient live at to provide possible medical service, after the service is done,the nurse record the emergency and reset the emergency through the patient terminal ,which will also be synced to the doctor workstation. If the nurse doesn't get the message on time, the workstation will report the situation to the aid center for help.

In the second circumstance, the physiological parameter such as blood pressure, blood oxygen and heart rate exceed the threshold set in the prescription, the alarm module will also intervene as following steps.

When the patient feel unwell and wears related sensors to measure the physiological parameter, or during the measurement the values exceed the thresholds, the terminal will detect this abnormal situation and connect to the doctor workstation to upgrade the physiological signals with a note representing the abnormality. The workstation will analyze the signals by the algorithms programmed in advance, if the situation is beyond algorithm's scope, the workstation will send the reminder message to the doctor for diagnose.

In the third circumstance, the system detect that probes of certain sensors fall off, the patient terminal will push notifications to the patient to put on the probes to the right place. If the patient doesn't operate for a certain period of time,the alarm system will start the alarm strategy just the same as mentioned in the previous circumstances.

\section{Conclusion and Future Works}

An doctor-patient interaction system for chronic disease telemonitoring is designed and implemented. The system consists of three modules with different functions which makes it easy to get essential medical information for both patients and doctors in chronic disease telemonitoring. With the system, both workloads and economic burdens can be reduced to a large scale.

As for future work, we will focus on the perfection of the system for specific medical needs, and will also concentrate on the automatic diagnosis algorithms to make it more intelligent thus further improve the efficiency of the system. 


\section{Acknowledgment}

This program is supported by the grants from the Initial Project for Post-Graduates of Hubei University of Medicine (2013QDJZR10), The author is very grateful for that.

\section{References}

[1]WHO Website. Available: http://www.who.int/topics/noncommunicable_diseases/en/

[2] D. L. Sackett, W. M. Rosenberg, J. A. Gray, R. B. Haynes, and W. S. Richardson, "Evidence based medicine: what it is and what it isn't," BMJ (Clinical research ed.), vol. 312, pp. 71-2, 1996-Jan-13 1996.

[3] E. H. Wagner, B. T. Austin, and M. Von Korff, "Organizing care for patients with chronic illness," The Milbank quarterly, vol. 74, pp. 511-44, 19961996.

[4] Y. H. Lin, I. C. Jan, P. C. I. Ko, Y. Y. Chen, J. M. Wong, and G. J. Jan, "A wireless PDA-based physiological monitoring system for patient transport," Ieee Transactions on Information Technology in Biomedicine, vol. 8, pp. 439-447, Dec 2004.

[5] W. Y. Chung, C. L. Yau, K. S. Shin, R. Myllyla, and Ieee, "A cell phone based health monitoring system with self analysis processor using wireless sensor network technology," in 2007 Annual International Conference of the Ieee Engineering in Medicine and Biology Society, Vols 1-16, ed, 2007, pp. 3705-3708.

[6] D. W. Curtis, E. J. Pino, J. M. Bailey, E. I. Shih, J. Waterman, S. A. Vinterbo, T. O. Stair, J. V. Guttag, R. A. Greenes, and L. Ohno-Machado, "SMART - An integrated wireless system for monitoring unattended patients," Journal of the American Medical Informatics Association, vol. 15, pp. 44-53, Jan-Feb 2008.

[7] K. A. Earle, R. S. H. Istepanian, K. Zitouni, A. Sungoor, and B. Tang, "Mobile Telemonitoring for Achieving Tighter Targets of Blood Pressure Control in Patients with Complicated Diabetes: A Pilot Study," Diabetes Technology \& Therapeutics, vol. 12, pp. 575-579, Jul 2010.

[8] Y. Tan, "Thinking in UML," ed: China WaterPower Press, 2012.

[9] J. Fawcett, Beginning XML,5th Edition: Tsinghua University press, 2013.

[10] G. Li, Crazy Android lecture: Electronic Industry Press, 2014.

[11]Android Developer Website. Available: http://www.androiddevtools.cn/ 